\title{
Long-term and low-thermal biological effects of microwaves
}

\author{
D. Adang ${ }^{1,2}$, C. Remacle ${ }^{3}$ \& A. Vander Vorst ${ }^{1}$ \\ ${ }^{1}$ EMIC Microwave Laboratory, Université Catholique de Louvain, \\ Louvain-la-Neuve, Belgium \\ ${ }^{2}$ ACOS Well-Being, Defence, Brussels, Belgium \\ ${ }^{3}$ BANI Biologie Animale, Université Catholique de Louvain, \\ Louvain-la-Neuve, Belgium
}

\begin{abstract}
There is evidence that radio frequencies $(\mathrm{RF})$ and microwaves directly affect living systems, as indicated by in vivo absorption experiments. Evidence is also provided by in vitro studies, revealing effects at various frequencies and intensities, on a number of cellular endpoints, including calcium binding, proliferation and alteration in membrane channels. There is ambiguity, however, about the relative contribution of direct and indirect non-thermal, i.e. lowthermal effects, as well as the possibility of direct low-thermal interactions. In this study, a possible causal link between microwave radiation (radar, cellular phone) and physiological and cellular changes is being evaluated by an epidemiological animal study on 128 rats, for 1.5 year. In order to assess the possible biological long-term effects of microwaves, we selected among others the following blood and hormonal parameters: lymphocytes, monocytes, granulocytes, erythrocytes, platelets, cytokines, corticosterone and ACTH (adrenocorticotrophic hormone). There is lack of knowledge about the biological mechanisms of the exposure to such low-level electromagnetic fields.

Keywords: electromagnetic fields, long-term biological effects, low-thermal, radar, cellular phone, microwaves, environmental risk.
\end{abstract}

\section{Introduction}

Radio frequencies (RF) and microwaves are non-ionizing, unlike much higher frequency waves above the visible range. The interaction of $\mathrm{RF} /$ microwaves with 
cell tissue can be considered as the result of three processes: penetration by electromagnetic waves and their propagation into the living system, primary interaction of the waves with cell tissue, and possible secondary effects of the primary interaction.

The word 'interaction' is important. It signals that end results depend not only on the action of the field but are influenced by the reaction of the living system. Living systems have a great capacity for compensating the effects induced by external influences, including electromagnetic sources. While it is often overlooked, it is one main reason why conclusions derived from computer models must be approached with caution. Guidelines for limiting the exposure provide protection against known adverse health effects; low-thermal long-term effects are not considered until today.

The thermal effects of microwaves are well known. There exists, however, a lot of uncertainties about the low-thermal long-term effects. This lack of scientific consensus led the World Health Organization to state that more scientific research is needed on this issue [1]. Very few people are exposed to thermally significant levels of microwaves. The big majority of exposures occur at levels at which weak-fields interactions would be the only possible source of any adverse health response. On the other hand, there is a major deficiency in the understanding of the effects of pulsed fields. Only a few isolated reports on pulsed fields are available while it is not possible to identify either the frequency or the peak power domains of importance [2]. Today, the general use of radar systems for navigation, together with the extension of radio communication links, satellite communications and cellular phone technology into the microwave frequency range, has implied a wider need for engineers and physicians to investigate the possible harmful effects of microwaves on the human environmental health. An increased or decreased presence of a biological marker does not necessarily indicate any pathological process, but may be a useful index for biological dosimetry of microwave exposure. The question of the microwave syndrome at low exposure level was raised several decades ago in Eastern Europe. It involves a number of signs like headache, perspiration, emotional instability, tiredness, somnolence, sexual problems, loss of memory, concentration and decision difficulties, insomnia, depressive hypochondriac tendencies, etc. Evaluation is difficult because of the absence of a control group and lack of reliable dosimetric data. A paper dated a few years ago supports RF sickness syndrome as a medical disorder [3].

\section{Objectives}

The objective of the present study consists in verifying the possible physiological modifications and anatomopathological changes due to low-level microwave exposure $(1 \mathrm{GHz}$ and $10 \mathrm{GHz})$, using both continuous and pulsed waves. In a first phase, this study focuses on selected blood and hormonal parameters as biological indicators of changes in the immune system of the animal. In a second phase, the possible carcinogenic effects will be investigated and microscopic study will be performed on all vital tissue. 


\section{Methods}

\subsection{An animal study}

There is an increasing concern about the possibility that radiofrequency exposure may play a role in the causation or promotion of cancer, especially related to the blood-forming organs [4].

A possible causal association between microwave exposure and biological effects of this kind of non-ionizing radiation is evaluated by an experimental study on a population of 128 Wistar albino rats, subdivided into 4 groups, depending on frequency and mode exposure, sham as well. To reduce as much as possible the stress factor, we have chosen for a collective exposure in an exposure unit, suitable for 32 rats, instead of an individual exposure where the rat is caged in a small waveguide. The different groups of rats are exposed two hours a day, seven days a week during eighteen months, to microwaves at $1 \mathrm{GHz}$ and $10 \mathrm{GHz}$, continuous and pulsed mode, at an average power density of 200 $\mu \mathrm{W} / \mathrm{cm}^{2}$ and $500 \mu \mathrm{W} / \mathrm{cm}^{2}$ respectively. This is explained in section 6 of this paper. In order to determine the possible biological long-term effects of microwaves, we selected the following parameters: blood cells, stress-induced hormones (adrenocorticotrophic hormone i.e. ACTH; corticosterone) and cytokines (interleukin i.e. IL-I, IL-X; tumor necrosis factor $\alpha$ i.e. TNF $\alpha$ ). Enzyme-linked immunosorbent assays are used to quantify those hormones in the blood plasma and serum. Body weight is monitored with minimal disturbance to the animal. It appears to be a reasonably sensitive indicator of stress, especially chronic stress, and therefore it is strongly recommended that animals which may be stressed should weighed regularly and their body weights compared with those of the sham exposed group. Daily observations of the rats' behaviour are part of the protocol.

\subsection{The exposure itself}

In a first series of experiments the Wistar albino rats are exposed in groups of 32 in an exposure unit $(1.11 \mathrm{~m} \times 0.60 \mathrm{~m} \times 0.71 \mathrm{~m})$, from the open end of a wave guide. This method constitutes an innovating element [5]. In most of the other studies indeed, rats are kept separately in plexiglass cylinders during exposure, where space to move is limited. Such narrow housing causes an enormous stress response in the rat [6]. This stressor creates a supplementary variable, which is susceptible to mask secretion of certain corticosteroids [7]. We use four-month old rats with a body weight of 300-350 grams. Four-month old rats are at the beginning of maturity and therefore ideal as a model for our experiments. We choose only male rats because of their constant metabolism compared to the variations in the female physiology. Animals are kept in normal vivarium conditions, and are made accustomed to experimental procedures, environment and handling two months prior to the beginning of the experiments. Daylight conditions start at $0700 \mathrm{a} . \mathrm{m}$. and darkness begins at $0900 \mathrm{p} . \mathrm{m}$. The Wistar albino 
rats are exposed to weak microwave doses as described above. It is important to run the experiment always on the same period of the day because of the influence of the circadian rhythm on the secretion of corticosteroids, for example corticosterone. Corticosterone is the most abundantly secreted glucocorticoid in the rat and helps the body to deal with stress. It acts as a negative feedback mechanism controlling the release of ACTH. It is therefore obviously important to sample glucocorticoids at the same time of the day if repeated measurements are to be made on different days. The peak in hormone secretion occurs towards the end of the light period. During the first months, the rats were already accustomed to manipulations and some blood samples were taken to assess a possible impact of a changing environment to the animal physiology. Handling, presumably acting as a mild stressor, during early life significantly reduced the glucocorticoid response to stress in adulthood [8].

\section{Materials}

The exposure unit is a self-made cage with the antenna - an open ended waveguide - on top (Figure 1). Its basis consists of a custom made box of polyethylene covered at the outside with microwave absorbing materials, themselves covered with wood (multiplex $15 \mathrm{~mm}$ ) to prevent any artefacts arising from objects introduced in the microwave field.

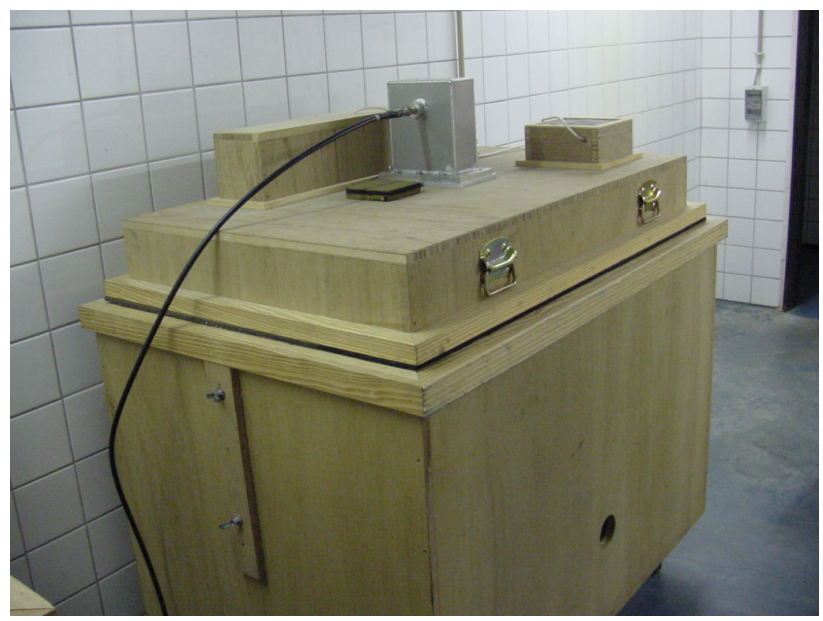

Figure 1: Collective exposure unit for rats.

The microwave generator used is a sweep oscillator Hewlett Packard 8620A. The cover of the box is also self-constructed and its height is $0.12 \mathrm{~m}$ to be sure that the rats are exposed to the far field of the antenna. A rectangular aperture is 
made, covered by a fine-meshed gauze, on which is fitted the armature of a fluorescent lamp. Furthermore, a fan is built in to improve the internal ventilation of the exposure unit.

\section{Rat marking}

To identity the rats during the whole experiment (one and a half year) in general and during blood sampling in particular, an unmistakable permanent method to distinguish one rat from another has been thought out. The ear of the rat is pierced following a formerly established pattern of figures. This procedure is carried out under total anaesthesia for one minute on the basis of sevoflurane.

\section{An appropriate exposure level}

Based on ICNIRP [9] recommendations, an exposure level is derived related to both frequencies to which the Wistar albino rats are submitted. The deposition of radio frequency energy in the human body tends to increase the body temperature. During exercise, the metabolic heat production can reach levels of $3-5 \mathrm{~W} / \mathrm{kg}$. In normal thermal environments, a specific absorption rate (SAR) of $1-4 \mathrm{~W} / \mathrm{kg}$ for 30 minutes produces average body temperature increases of less than $1{ }^{\circ} \mathrm{C}$ for healthy adults. Thus an occupational radio frequency guideline of $0.4 \mathrm{~W} / \mathrm{kg}$ SAR leaves a margin of protection against complications to the thermally unfavourable environmental conditions. For the general population, which includes sensitive subpopulations, such as children and elderly, a SAR of $0.08 \mathrm{~W} / \mathrm{kg}$ would provide a further margin of safety against adverse thermal effects from radio frequency fields. That is why one has defined basic restrictions based on observed biological effects. Assuming that the ratio in size between a human and a rat equals 10, one has to 'adapt' the ICNIRP recommendations, valuable for a human being, to the size of the rat by multiplying by 10 the key frequencies. This means that the exposure of a rat of $1 \mathrm{GHz}$ corresponds to a human exposure of $100 \mathrm{MHz}$. In other words, an exposure of $1 \mathrm{GHz}$ for a rat is equivalent of a $100 \mathrm{MHz}$ exposure for man. The basis restriction value for man at $100 \mathrm{MHz}$ is $2 \mathrm{~W} / \mathrm{m}^{2}$. This means that a power density of $200 \mu \mathrm{W} / \mathrm{cm}^{2}$ is suitable in relationship with the exposure of rats. Following an identical reasoning leads us to the fact that a $1 \mathrm{GHz}$ exposure for man corresponds to a $10 \mathrm{GHz}$ exposure for rats. ICNIRP recommendations stipulate a basic restriction, expressed in power density, of $\mathrm{f} / 200(\mathrm{f}=$ frequency), i.e. $1000 \mathrm{MHz} / 200$ or $5 \mathrm{~W} / \mathrm{m}^{2}\left(500 \mu \mathrm{W} / \mathrm{cm}^{2}\right)$. In the far field, $200 \mu \mathrm{W} / \mathrm{cm}^{2}$ corresponds to an electric field strength of $27 \mathrm{~V} / \mathrm{m}$. That's the reason why we focus on this value in the next paragraph.

\section{Results of the field uniformity measurements at $1 \mathrm{GHz}$}

The evaluation of the field uniformity is carried out by means of an electric field measurement, using the Isotropic Field Monitor of the Company Amplifier Research. 
Table 1: $\quad$ Measured electric field $\mathrm{E}$ and power density $\mathrm{P}$.

\begin{tabular}{|c|c|c|c|c|c|}
\hline Square nr. & $\mathrm{E}[\mathrm{V} / \mathrm{m}]$ & $\mathrm{P}\left[\mu \mathrm{W} / \mathrm{cm}^{2}\right]$ & Square nr. & $\mathrm{E}[\mathrm{V} / \mathrm{m}]$ & $\mathrm{P}\left[\mu \mathrm{W} / \mathrm{cm}^{2}\right]$ \\
\hline A1 & 26.5 & 186.3 & D7 & 27.2 & 196.2 \\
\hline A2 & 25.4 & 171.1 & D8 & 21.5 & 122.6 \\
\hline A3 & 24.0 & 151.0 & D9 & 23.5 & 146.5 \\
\hline A4 & 20.2 & 108.2 & D10 & 32.4 & 278.5 \\
\hline A5 & 29.0 & 223.1 & D11 & 18.7 & 92.7 \\
\hline A6 & 32.3 & 276.7 & E1 & 31.4 & 261.5 \\
\hline A7 & 25.2 & 168.4 & E2 & 19.4 & 99.8 \\
\hline A8 & 34.0 & 306.6 & E3 & 26.7 & 189.1 \\
\hline A9 & 36.3 & 349.5 & E4 & 28.5 & 215.5 \\
\hline A10 & 30.2 & 241.9 & E5 & 8.8 & 20.5 \\
\hline A11 & 24.2 & 155.3 & E6 & 31.4 & 261.5 \\
\hline B1 & 32.8 & 285.4 & E7 & 25.2 & 168.4 \\
\hline B2 & 27.2 & 196.2 & E8 & 22.2 & 130.7 \\
\hline B3 & 14.4 & 55.0 & E9 & 28.7 & 217.0 \\
\hline B4 & 22.2 & 130.7 & E10 & 36.0 & 343.8 \\
\hline B5 & 25.4 & 171.1 & E11 & 20.9 & 115.9 \\
\hline B6 & 35.6 & 336.2 & F1 & 34.8 & 321.2 \\
\hline B7 & 28.4 & 213.9 & F2 & 25.5 & 172.5 \\
\hline B8 & 37.3 & 369.0 & F3 & 17.7 & 83.1 \\
\hline B9 & 49.2 & 642.1 & F4 & 21.8 & 126.1 \\
\hline B10 & 34.0 & 306.5 & F5 & 13.6 & 49.1 \\
\hline B11 & 43.0 & 490.5 & F6 & 33.6 & 299.5 \\
\hline $\mathrm{C} 1$ & 32.4 & 278.5 & F7 & 29.5 & 230.8 \\
\hline $\mathrm{C} 2$ & 24.6 & 160.5 & F8 & 31.2 & 258.2 \\
\hline $\mathrm{C} 3$ & 8.2 & 17.8 & F9 & 35.4 & 332.4 \\
\hline $\mathrm{C} 4$ & 20.7 & 113.7 & F10 & 36.8 & 359.3 \\
\hline $\mathrm{C} 5$ & 13.6 & 49.1 & F11 & 38.6 & 395.2 \\
\hline C6 & 29.7 & 234.0 & $\overline{\text { G1 }}$ & 30.6 & 248.4 \\
\hline $\mathrm{C} 7$ & 26.7 & 189.1 & $\mathrm{G} 2$ & 22.8 & 137.9 \\
\hline $\mathrm{C} 8$ & 21.0 & 117.0 & G3 & 25.9 & 177.9 \\
\hline C9 & 36.6 & 355.3 & G4 & 23.2 & 142.8 \\
\hline $\mathrm{C} 10$ & 34.9 & 323.1 & G5 & 29.3 & 227.7 \\
\hline C11 & 23.0 & 140.3 & G6 & 41.8 & 463.5 \\
\hline D1 & 35.8 & 340.0 & G7 & 32.8 & 285.4 \\
\hline $\mathrm{D} 2$ & 44.8 & 532.4 & G8 & 30.4 & 245.1 \\
\hline D3 & 25.4 & 171.1 & G9 & 32.2 & 275.0 \\
\hline D4 & 21.4 & 212.5 & G10 & 23.0 & 140.3 \\
\hline D5 & 12.0 & 38.2 & G11 & 25.0 & 165.8 \\
\hline D6 & 28.5 & 215.4 & & & \\
\hline
\end{tabular}

\begin{tabular}{|c|c|c|}
\hline & $\mathrm{E}[\mathrm{V} / \mathrm{m}]$ & $\mathrm{P}\left[\mu \mathrm{W} / \mathrm{cm}^{2}\right]$ \\
\hline Mean & 27.2 & 201.2 \\
\hline SD & 7.4 & 114.6 \\
\hline
\end{tabular}


This instrument measures the electric field $\mathrm{E}$ in space along three axes. Afterwards, the corresponding power density values were derived from these data. The bottom of the exposure unit is first subdivided in 77 squares of $10 \mathrm{~cm}^{2}$. In a following step the probe is placed successively in the center of each square and the electric field strength is recorded. The squares are denominated from [AG][1-11]. The mean value of the electric field strength is $27 \mathrm{~V} / \mathrm{m}$ (standard deviation $10 \mathrm{~V} / \mathrm{m}$ ). In order to make the electric field more uniform some PVC constructions are build at the inside of the exposure unit. We started from the rough data of the measurement series and plotted them in a scheme of the exposure unit. Afterwards we focused on the squares were the value of the electric field is less than $27 \mathrm{~V} / \mathrm{m}$. Empirically, we constructed on the bottom of the exposure unit a PVC platform-like shape, to uniformize the electric field followed by an analogous measurement session as mentioned above. These constructions also reduce the stress level of the rats. The results are shown in Table 1.

\section{Results}

Base line determination blood samples have been collected early March. In the meantime, statistical analysis of 10000 blood parameters is in progress.

Preliminary results will be presented at the Environmental Health Risk 2005 Conference.

\section{References}

[1] M. Repacholi, Low-level exposure to radio frequency electromagnetic fields: health effects and research needs, Bioelectromagnetics, 19 (1), pp. 1-19, 1998.

[2] A. Laurence et al. Biological effects of electromagnetic fields-mechanisms for the effects of pulsed microwave radiation on protein conformation, J. Ther. Biol., 206, pp. 291-298, 2000.

[3] A.G. Johnson Liakouris, Radiofrequency (RF) sickness in the Lelienfield study: an effect of modulated microwaves?, Arch. Environm. Health, 53(3), pp. 236-238, May-June 1998.

[4] H. Yoav, The potential carcinogenic hazards of electromagnetic radiation: a review, Cancer Detection and Prevention, 19(3), pp. 244-249, 1959.

[5] D. Adang, Study on the long-term athermal biological effects of radar radiation in rats, Proceedings of URSI Forum 2003, Brussels, December, 2003.

[6] K. Gärtner et al., Stress response of rats to handling and experimental procedures, Lab. Anim., 14, pp. 267-274, 1980.

[7] C.E. Manser, The assessment of stress in laboratory animals, Royal Society for the Prevention of Cruelty to Animals, RSPCA, London, UK, pp. 30-32, 1992. 
404 Environmental Health Risk II

[8] A. Armario, J.L. Montero, J. Balash, Sensitivity of corticosterone and some metabolic variables to graded levels of low intensity stresses in adult male rats, Phys. Behav., 37, pp. 595-561, 1986.

[9] ICNIRP Guidelines, Guidelines for limiting exposure to time-varying electric, magnetic and electromagnetic fields (up to $300 \mathrm{GHz}$ ), Health Physics, vol. 74, pp. 494-522, April 1998. 